\title{
Plastic stress intensity factor behavior at small and large scale yielding
}

\author{
Alexander Zakharov, Valery Shlyannikov, Anastasia Tartygasheva \\ Institute of Power Engineering and Advanced Technologies, FRC Kazan Scientific Center, Russian Academy of Sciences \\ alex.2akharov88@mail.ru,shlyannikov@mail.ru,tartigashevaa@mail.ru
}

\begin{abstract}
In this paper the plastic stress intensity factor (SIF) is used to study the coupling effects of the loading biaxiality, the material properties and the cracked body configuration in both the small- and large-scale yielding ranges. A finite element (FE) analysis is performed for a cracked Mode I plane strain plate subjected to biaxial tension/compression loading. The governing parameter of the elastic-plastic crack-tip stress field $I_{n}$-integral at the crack tip, the J-integral, and the plastic SIF, are calculated as a functions of the loading biaxiality and the applied stress levels. The different trend of the $I_{n}$-integral distributions as a function of the applied stresses with respect to the $J$-integral is demonstrated. The contrary character of the distributions of the plastic SIF and the $J$-integral as a function of the biaxial stress ratio is observed.

Special emphasis is put on the behavior of the $J$-integral and the plastic SIF for the specified test specimen geometries under mixed mode loading. The coupling effects of the mixed mode fracture and the material properties on the $J$ integral and the plastic SIF distributions for a set of the specimen configurations are stated. Comparative analysis of the values of the plastic SIF calculated for both small-scale and large-scale yielding in the test specimen configurations considered here is presented. A significant difference between the small- and the large-scale yielding plastic SIF in the full range of mixed modes is shown.
\end{abstract}

KEYWORDS. Biaxial loading; J-integral; Plastic stress intensity factor; Mixed mode loading.

\section{open Access}

Citation: Zakharov A., Shlyannikov V.., Tartygasheva A., Plastic stress intensity factor behavior at small and large scale yielding, Frattura ed Integrità Strutturale, 53 (2020) 223-235.

Received: 14.04 .2020

Accepted: 06.05.2020

Published: 01.07.2020

Copyright: (C) 2020 This is an open access article under the terms of the CC-BY 4.0, which permits unrestricted use, distribution, and reproduction in any medium, provided the original author and source are credited.

\section{INTRODUCTION}

A ccording to the Hutchinson's analytical solution [1], the near-tip field at a stationary Mode I crack for an elasticplastic power hardening material is governed by the plastic SIF which plays a role analogous to that of elastic SIF and it is directly related to $J$-integral by a simple equation for small-scale yielding conditions. Shih [2] extended the HRR solution for the case of small-scale yielding mixed-mode fracture and showed that an important feature of such analysis is the formulation of the additional parameter governing the singular stress fields for an elastic-plastic material. Shlyannikov and Tumanov [3] reconsidered the Hutchinson [1] and Shih [2] solutions, for both pure Mode I and mixed mode conditions 
respectively, and proposed a new method for analytical solution of plane mixed mode problems. Shlyannikov et al. [4-6] demonstrated the application of the plastic SIF as a measure of the fracture resistance characteristics under monotonic static and cyclic loading.

It is well known that a small-scale yielding analysis of the fracture is usually considered applicable for investigating the plastic zone size at the crack tip; for example, for an infinite cracked plate, less than one order of the crack length and dimensionless nominal stress $\bar{\sigma}_{n}=\sigma_{n} / \sigma_{0}$ normalised by the yield stress up to $\sim 0.2$. However, at the higher values of the applied nominal stresses, the plastic zone is no longer small compared to the crack length and the plastic SIF required some modification. For large-scale yielding, the J-integral cannot be calculated simply, in general, since it depends on the geometry, the load level and the nonlinear stress-strain behavior. Hilton and Hutchinson [7], Hilton and Sih [8], and Hilton [9] investigated the behaviour of the plastic stress or strain intensity factor, for both the small- and large-scale yielding ranges. Hilton [7] and Lee and Liebowitz [10] studied the biaxial loading effects on the plastic stress and strain intensity factor behaviour for pure Mode I cracked-plate problems and showed that the biaxial effects on nonlinear fracture resistance parameters increase as the applied nominal stress increases. Lee and Liebowitz's proposed an algorithm for numerical determination of the $J$ integral under large-scale yielding conditions to avoid increasing the differences in the values of the plastic SIF in a full range of biaxial nonlinear deformation.

In this paper the comparative analysis of the plastic SIF behaviour for the different cracked body configurations in both the small- and large-scale yielding ranges is presented. FE analysis was performed for the cracked Mode I plane strain plate subjected to biaxial tension/compression loading. The governing parameter of the elastic-plastic crack-tip stress field $I_{n}$ factor at the crack tip, the $J$-integral, and the plastic SIF, were calculated as a functions of loading biaxiality and the applied stress levels. Special emphasis was put on the behavior of $J$-integral and the plastic SIF for the specified test specimen geometries under mixed mode loading.

\section{OBJECTS OF STUDY AND LOADING CONDITIONS}

S ubjects of the current study are an infinite size center-cracked plate (CCP) under biaxial stress fields and two types of cruciform specimen and compact tension-shear specimen under mixed mode loading. In the first part of this paper, the plastic SIF was employed to study the coupling effects of nominal stress level and loading biaxiality for CCP in both the small- and large-scale yielding ranges by means of the plane strain and 3D nonlinear FE analyses. A rectangular plate of width $2 w$ with a central crack of length $2 a$ subjected to the different types of biaxial loading is presented in Fig. 1.

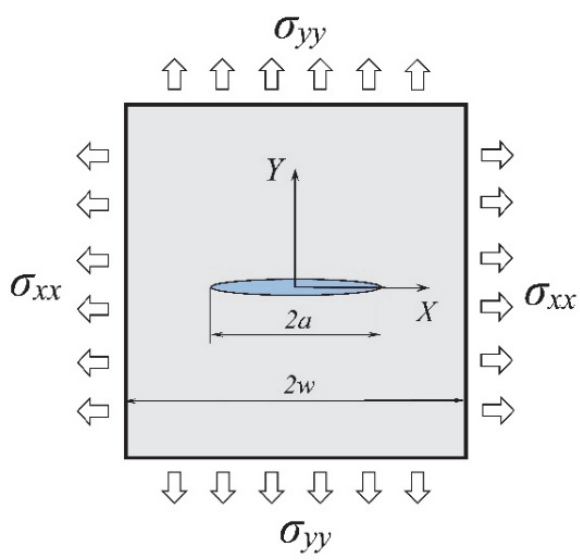

Figure 1: The Center-cracked plate under biaxial loading.

The center-cracked plate could be subjected to two perpendicular loads: one parallel to the Y-axis $\sigma_{y y}^{\infty}$ and another parallel to the X-axis $\sigma_{x x}^{\infty}$. The magnitude of the traverse load $\sigma_{x x}^{\infty}$ is described by the nominal stresses biaxial ratio $\eta=\sigma_{x x}^{\infty} / \sigma_{y y}^{\infty}$ . The different degrees of biaxial nominal stress ratio were considered from equibiaxial tension $(\eta=+1)$ up to equibiaxial tension-compression $(\eta=-1)$. The Ti6Al4V titanium alloy was identified as a material of the CCP.

The main mechanical properties and elastic-plastic parameters describing the nonlinear behavior of the materials have been determined by the standard tension tests according to the ASTM E8 standard [11]. The cylindrical smooth specimens were 
tested at room temperature. Stress-strain curve was described by the well-known Ramberg-Osgood equation in the following form:

$$
\varepsilon=\frac{\sigma}{E}+\alpha \frac{\sigma_{0}}{E}\left(\frac{\sigma}{\sigma_{0}}\right)^{n}
$$

where $\varepsilon$ is the strain, $\sigma$ is the stress, $\sigma_{0}$ is the yield stress, $E$ is the Young's modulus, $n$ is the strain hardening exponent and $\alpha$ is the strain hardening coefficient of the Ramberg-Osgood model.

As a result, the main mechanical properties were determined for the Ti6Al4V: namely, the Young's modulus $E=118000$ $\mathrm{MPa}$; the Poisson's ratio $v=0.3$; the yield stress $\sigma_{0}=885 \mathrm{MPa}$; the ultimate stress $\sigma_{u}=1289 \mathrm{MPa}$; the strain hardening exponent $n=12.59$ and the strain hardening coefficient $\alpha=1.225$.

As it mentioned before, in this paper special emphasis was put on the behavior of the J-integral and the plastic SIF for the specified test specimen geometries under mixed mode loading. Configurations of the flat cruciform specimen (CS-1), the cruciform specimen with thinned working area (CS-2) and the compact tension-shear specimen (CTS) are presented in Fig.2.

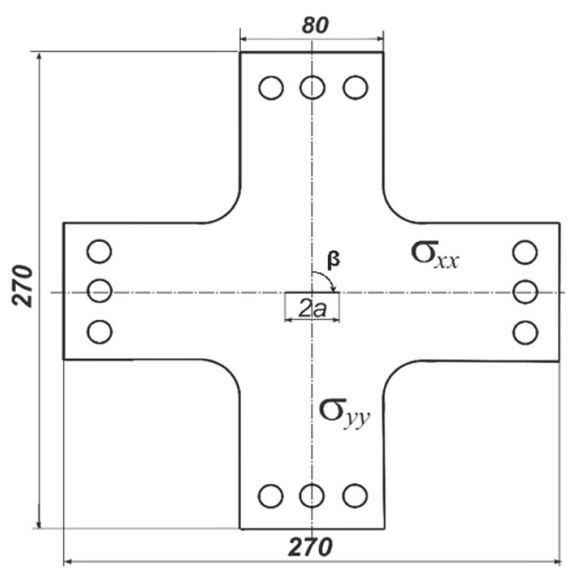

a)

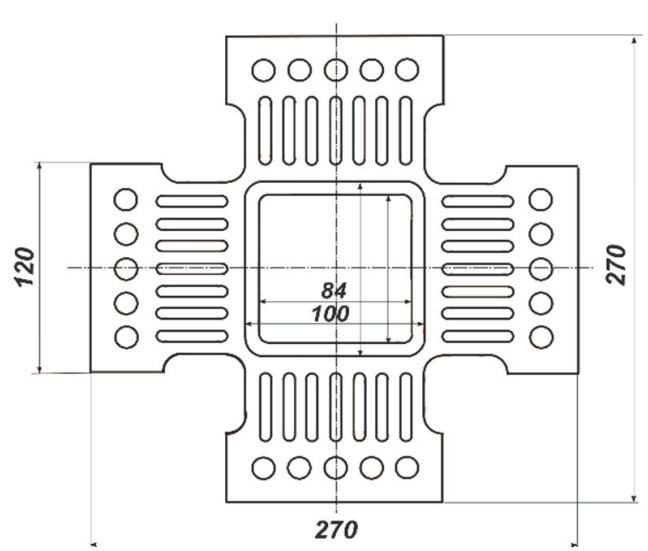

b)
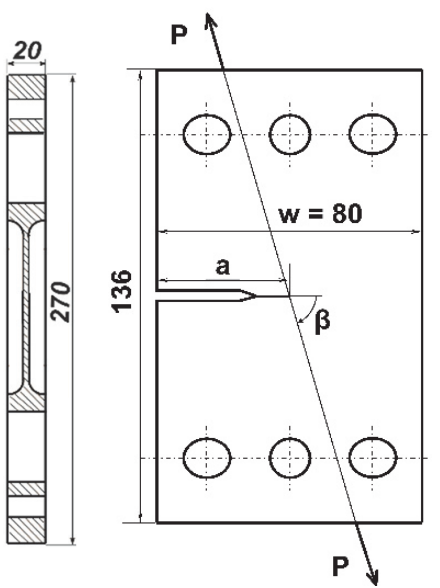

c)

Figure 2: Test specimen configurations: (a) the flat cruciform specimen, (b) the cruciform specimen with thinned working area, (c) the compact tension-shear specimen.

The different degrees of mode mixity from pure Mode I to pure Mode II can be realized in all specimens by the combinations of the nominal stress level $\sigma_{n}$, the remote biaxial stress ratio $\eta=\sigma_{x x} / \sigma_{y y}$ and the initial crack angle $\beta$ with respect to the loading direction. For the biaxial loaded cruciform specimens, $\beta=90^{\circ}$, correspond to pure Mode I, whereas pure Mode II can be realized when $\beta=45^{\circ}$ and $\eta=-1$. In the CTS $\beta=90^{\circ}$ corresponds to pure Mode I, and pure Mode II can be achieved when $\beta=0^{\circ}$. FE-analyzes of the specified test specimen geometries were performed for the two types of steels and the titanium and aluminum alloys with different elastic-plastic properties. Similarly, to the Ti6Al4V titanium alloy, the main mechanical properties for the other materials were determined by the standard tension tests at room temperature. The main mechanical properties of the considered materials are listed in Tab. 1.

\begin{tabular}{cccccc}
\hline Material & $\begin{array}{c}\text { Young's } \\
\text { modulus, } E \\
(\mathrm{MPa})\end{array}$ & $\begin{array}{c}\text { Yield stress, } \\
\sigma_{0}(\mathrm{MPa})\end{array}$ & $\begin{array}{c}\text { Ultimate } \\
\text { stress, } \sigma_{f} \\
(\mathrm{MPa})\end{array}$ & $\begin{array}{c}\text { Strain } \\
\text { hardening } \\
\text { exponent, } n\end{array}$ & $\begin{array}{c}\text { Strain } \\
\text { hardening } \\
\text { coefficient, } a\end{array}$ \\
Steel R2M & 226900 & 362.4 & 1190 & 4.141 & 4.131 \\
Steel 34CrN3MA & 216210 & 714.4 & 1260.4 & 7.889 & 0.529 \\
Ti6Al4V & 70570 & 471.6 & 700 & 10.851 & 1.570 \\
\hline
\end{tabular}

Table 1: The main mechanical properties. 


\section{PLASTIC STRESS INTENSITY FACTOR FOR SMALL- AND LARGE-SCALE YIELDING}

\section{Small scale yielding}

$\mathrm{t}$ the small scale yielding the plastic SIF $K_{P}$ in pure Mode I (or pure Mode II) can be expressed directly in terms of a corresponding elastic stress intensity factor using Rice's J-integral [12]. For an infinite plate with a centred line crack subjected to mixed-mode loading, the plastic SIF $K_{P}$ and the J-integral are related by the Shih's relation [2]:

$$
J=\frac{K_{1}^{2}}{E}=\frac{\bar{\alpha} \sigma_{0}^{2}}{E} I_{n}\left(K_{p}\right)^{n+1}
$$

where the components of the $J$-integral are defined in terms of elastic stress intensity factors $K_{1}$ and $K_{2}$ by:

$$
\begin{aligned}
& J_{x}=\frac{(1+v)(1+\kappa)}{4 E}\left(K_{1}^{2}+K_{2}^{2}\right) \\
& J_{y}=-\frac{2(1+v)(1+\kappa)}{4 E} K_{1} K_{2}
\end{aligned}
$$

Where $E$ is the Young's modulus, $v$ is the Poisson's ratio, $k=3-4 v$. Substituting Eqs. (3) and (4) into Eqn. (2) leads to the following expression:

$$
K_{P}=\left[\frac{(1+v)(1+\kappa)}{4 \alpha I_{n} w} \frac{\sqrt{\left(K_{1}^{2}+K_{2}^{2}\right)^{2}+4\left(K_{1} K_{2}\right)^{2}}}{\sigma_{0}^{2}}\right]^{\frac{1}{n+1}}
$$

The elastic SIF for a finite size cracked specimen under mixed mode loading conditions can be expressed as:

$$
\begin{aligned}
& K_{1}=\frac{\sigma \sqrt{\pi a}}{2}[1+\eta-(1-\eta) \cos 2 \beta] Y_{1}\left(\frac{a}{w}, \beta, T\right) \\
& K_{2}=\frac{\sigma \sqrt{\pi a}}{2}[(1-\eta) \sin 2 \beta] Y_{2}\left(\frac{a}{w}, \beta, T\right)
\end{aligned}
$$

where $a$ is the crack length, $\beta$ is the crack angle, $\sigma_{n}$ is the nominal applied stress, $\sigma_{0}$ is the yield stress, $\eta$ is the load biaxiality ratio, $w$ is the specimen width, $T$ is the nonsingular stress, $Y_{i}(a / w, \beta, T)$ are the geometry dependent correction factors.

The governing parameter of the crack-tip elastic-plastic stress-strain field in the form of the $I_{n}$-integral in Eqn.2 and Eqn.5 is a function of the material strain hardening exponent $n$ and the angular stress/displacement distributions. Shlyannikov and Tumanov [3] suggested the numerical procedure for calculating $I_{n}$-integral for the different cracked bodies by means of the elastic-plastic FE-analysis of the near crack-tip stress-strain fields: [2]:

$$
\begin{aligned}
& I_{n}^{F E M}\left(\theta, M_{p}, n,(a / w)\right)=\int_{-\pi}^{+\pi} \frac{n}{n+1}\left(\tilde{\sigma}_{e}^{n+1}\right)^{F E M} \cos \theta- \\
& -\left[\tilde{\sigma}_{r r}^{F E M}\left(\tilde{u}_{\theta}^{F E M}-\frac{d \tilde{u}_{r}^{F E M}}{d \theta}\right)-\tilde{\sigma}_{r \theta}^{F E M}\left(\tilde{u}_{r}^{F E M}+\frac{d \tilde{u}_{\theta}^{F E M}}{d \theta}\right)\right] \sin \theta- \\
& -\frac{1}{n+1}\left(\tilde{\sigma}_{r r}^{F E M} \tilde{u}_{r}^{F E M}+\tilde{\sigma}_{r \theta}^{F E M} \tilde{u}_{\theta}^{F E M}\right) \cos \theta .
\end{aligned}
$$


Eqn. (8) was used to determine the plastic SIF for the cracked plate in the full range loading biaxiality and both the smallscale and large scale plasticity.

\section{Large scale yielding}

There are several possible ways to use fully plastic strain hardening solutions to determine the behaviour at large-scale yielding, based on realistic tensile stress-strain curves involving both elastic and plastic parts of the strain energy density [710]. The method proposed by Lee and Liebowitz [10] for the elastic-plastic solids is one of them. The J-integral for the large-scale yielding conditions can be expressed in the following form:

$$
\bar{J}_{f}=\frac{\sigma_{0}^{2} w}{E} \int_{\Gamma}\left(\bar{W}_{f} d y-\bar{\sigma}_{i j} n_{j} \bar{u}_{i, x} d s\right)
$$

where $\Gamma$ is a curve that surrounds the crack tip, starting from the lower crack flank, traversing counterclockwise, and ending on the upper crack flank; $s$ is the arc length; $n_{i}$ is the outward unit vector normal to the curve; and $\bar{u}_{i}$ is the dimensionless displacement. The dimensionless strain energy density in the formula ( 9 ) is described by the following equation:

$$
\bar{W}_{f}=\left[\frac{1+v}{3} \bar{\sigma}_{e}^{2}+\frac{1-2 v}{6} \bar{\sigma}_{k e}^{2}+\frac{\bar{\alpha} n}{n+1} \bar{\sigma}_{e}^{n+1}\right]
$$

In Eqn. (10), the stress tensor and invariant are both normalised by the yield stress: $\bar{\sigma}_{i j}=\sigma_{i j} / \sigma_{0}$ and $\bar{\sigma}_{k k}=\sigma_{k k} / \sigma_{0}$. Substituting Eqn.(10) into Eqn.(9) gives expression for the $J$-integral as the dimensionless stress and the displacements angular functions accounting for their derivatives with respect to the polar angle:

$$
\begin{aligned}
& J_{f}^{F E M}=\frac{r \sigma_{0}^{2}}{E} \int_{-\pi}^{+\pi}\left[\frac{1+v}{3} \bar{\sigma}_{e}^{2}+\frac{1-2 v}{6} \bar{\sigma}_{k k}^{2}+\frac{\bar{\alpha} n}{n+1} \bar{\sigma}_{e}^{n+1}\right] \cos \theta d \theta- \\
& -r \sigma_{0}^{2} \int_{-\pi}^{+\pi}\left(\bar{\sigma}_{r r} \frac{\partial \bar{u}_{r}}{\partial r}+\bar{\sigma}_{r \theta} \frac{\partial \bar{u}_{\theta}}{\partial r}\right) \cos \theta d \theta+\sigma_{0} \int_{-\pi}^{+\pi}\left(\bar{\sigma}_{r r} \frac{\partial \bar{u}_{r}}{\partial \theta}+\bar{\sigma}_{r \theta} \frac{\partial \bar{u}_{\theta}}{\partial \theta}\right) \sin \theta d \theta
\end{aligned}
$$

where:

$$
\begin{aligned}
& \frac{\partial}{\partial x}=\frac{\partial}{\partial r} \cos \theta-\frac{\sin \theta}{r} \frac{\partial}{\partial \theta} \\
& \frac{\partial}{\partial y}=\frac{\partial}{\partial r} \sin \theta+\frac{\cos \theta}{r} \frac{\partial}{\partial \theta} \\
& d y=r \cos \theta, d S=r d \theta
\end{aligned}
$$

Given the J-integral formulation for large-scale yielding in the form of Eqn. (9) the expression for the plastic stress intensity factor can be written in the following form:

$$
\bar{K}_{P}=\left(\frac{\bar{J}_{f}}{\bar{\alpha} I_{n}}\right)^{\frac{1}{n+1}}, \bar{J}_{f}^{F E M}=J_{f}^{F E M}\left(\frac{E}{w \sigma_{0}^{2}}\right)
$$

where the $I_{n}$-integral is defined by Eqn. (8).

Notice that the stresses and the displacements components in Eqs. (8) and (11) were obtained through the FE analysis of the near crack tip stress-strain fields. 


\section{SMALL-AND LARGE-SCALE YIELDING ANALYSIS OF CENTRAL CRACKED PLATE UNDER BIAXIAL LOADING}

$\mathrm{T}$

his section of the current study is concerned with the numerical calculations and the results for the CCP subjected to biaxial loading. The plane strain nonlinear FE analysis were performed using ANSYS Code [13] for studying the coupling effects of loading biaxiality and level of the applied nominal stresses on the elastic-plastic crack-tip stress fields. The typical FE mesh for the CCP is illustrated in Fig.3a. In the case of plane strain problem the 2D eight-node isoparametric elements were used to the CCP FE model.

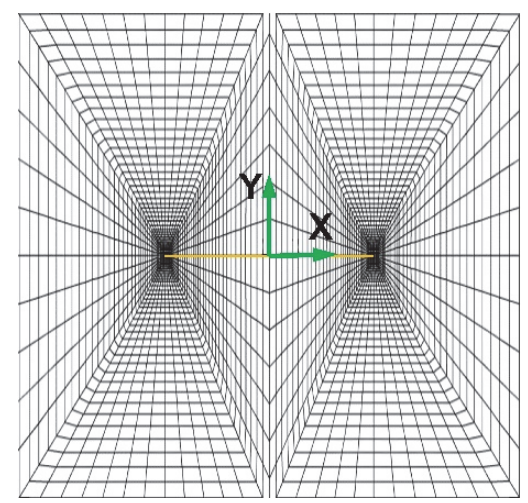

a)

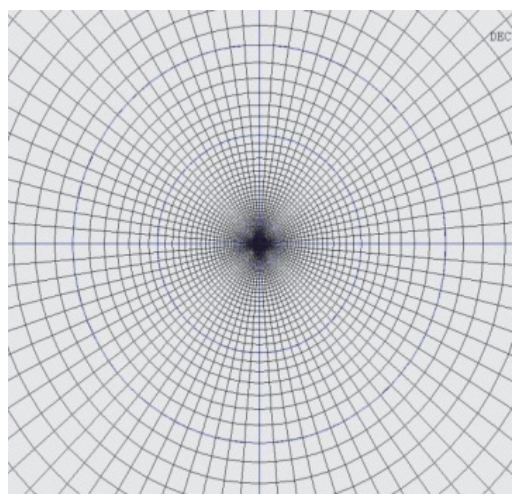

b)

Figure 3: The FE model for the center-cracked plate under biaxial loading and the finite elements at the crack tip region.

The FE mesh at the crack-tip region is presented in Fig.3b. Since the crack-tip region contains steep displacement and high stress gradients, the mesh needs to be very refined at the crack tip. For this purpose, a corresponding mesh topology having a focused ring of the elements surrounding the crack front was used to enhance convergence of the numerical solutions. For CCP at the crack tip area in the circumferential direction, 40 equally sized elements are defined in the angular region from 0 to $\pi$. The size of each ring increases gradually with the radial distance from the crack tip. The order of magnitude of the smallest element size close to near the crack tip is equal to $10^{-6} \mathrm{~m}$. For all elastic-plastic FE analyzes the CCP with mathematical notch type crack when the radius at the crack tip equal to zero was considered.

The numerical results for the CCP were presented for the Mode I plane strain conditions. The $J$-integral, the governing parameter of the elastic-plastic crack-tip stress field the $I_{n}$-integral and the plastic SIF were obtained as a functions of the loading biaxiality and the applied stress levels. Fig.4 shows the numerical results of the $J$-integral calculations for the CCP from the titanium alloy Ti6A14V under different types of the biaxial loading as a function of the applied stress levels. The $J$ integral distributions were obtained for two crack -tip distances normalized by the crack length $\bar{r}=r / a$. For all the biaxial nominal stress ratios considered here the $J$-integral distributions have the same trend and it is an increasing function of the applied stress levels. It should be noted that the $J$-integral is almost unchanged for both the crack-tip distances when the applied stress level $\sigma / \sigma_{0} \leq 0.15$. However, in the case with an applied stress level when $\sigma / \sigma_{0}$ higher than 0.15 the $J$-integral distributions differ increasingly as a function of the biaxial stress ratio.

The distributions of the governing parameter of the elastic-plastic crack-tip stress $I_{n}$-integral as a function of the applied stress levels in the full range of the biaxial stress ratio are plotted in Fig.5. Opposite trend of the $I_{n}$-integral distributions as a function of the applied stresses with respect to the J-integral is observed. The $I_{n}$-integral is decreased with increasing the applied stresses. It is founded that for both the crack-tip distances the $I_{n}$-integral is very sensitive to the type of the biaxial stress state in the full range of the applied stress levels. As the biaxial stress ratio varies from the equibiaxial tension $(\eta=$ $+1)$ to the equibiaxial tension-compression $(\eta=-1)$ values of the $I_{n}$-integral are decreased. It can be concluded, that the $I_{n^{-}}$ integral values are very sensitive to the type of the biaxial loading, therefore the plastic SIF also clearly depends on the biaxial loading conditions. The character of the $I_{n}$-integral distributions as a function of the biaxial loading is depended on the crack-tip distance.

Fig.6 shows the $J$-integral distributions as a function of the biaxial stress ratio in the range of considered the applied stress levels. As it follows from these results for both the crack-tip distances the type of the biaxial loading have not effect on the $J$-integral distributions when the applied stresses $\sigma / \sigma_{0} \leq 0.15$. In the range of the applied stresses $\sigma / \sigma_{0}$ higher than 0.15 the $J$-integral values under the equibiaxial tension-compression $(\eta=-1)$ are lower than $J$-integral at the equibiaxial tension $(\eta=$ $+1)$. 

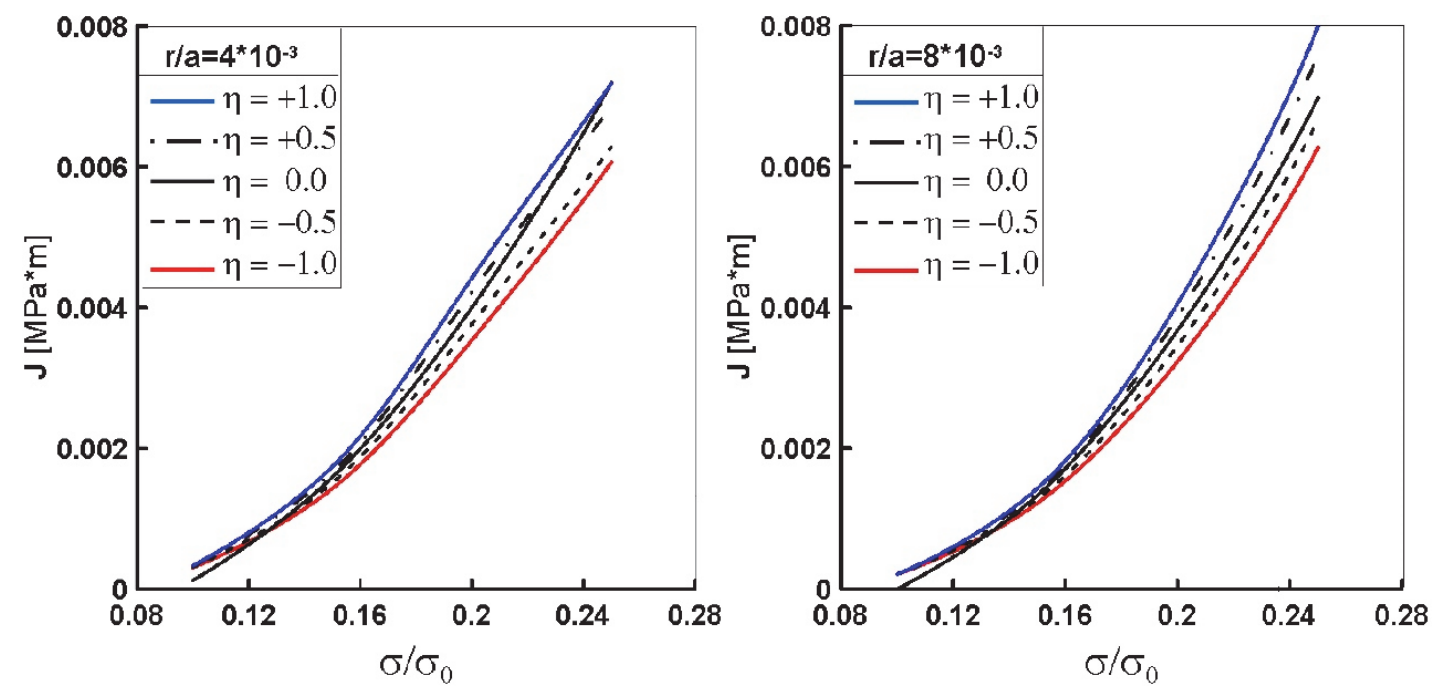

Figure 4: The $J$-integral distributions as a function of the applied stress level.
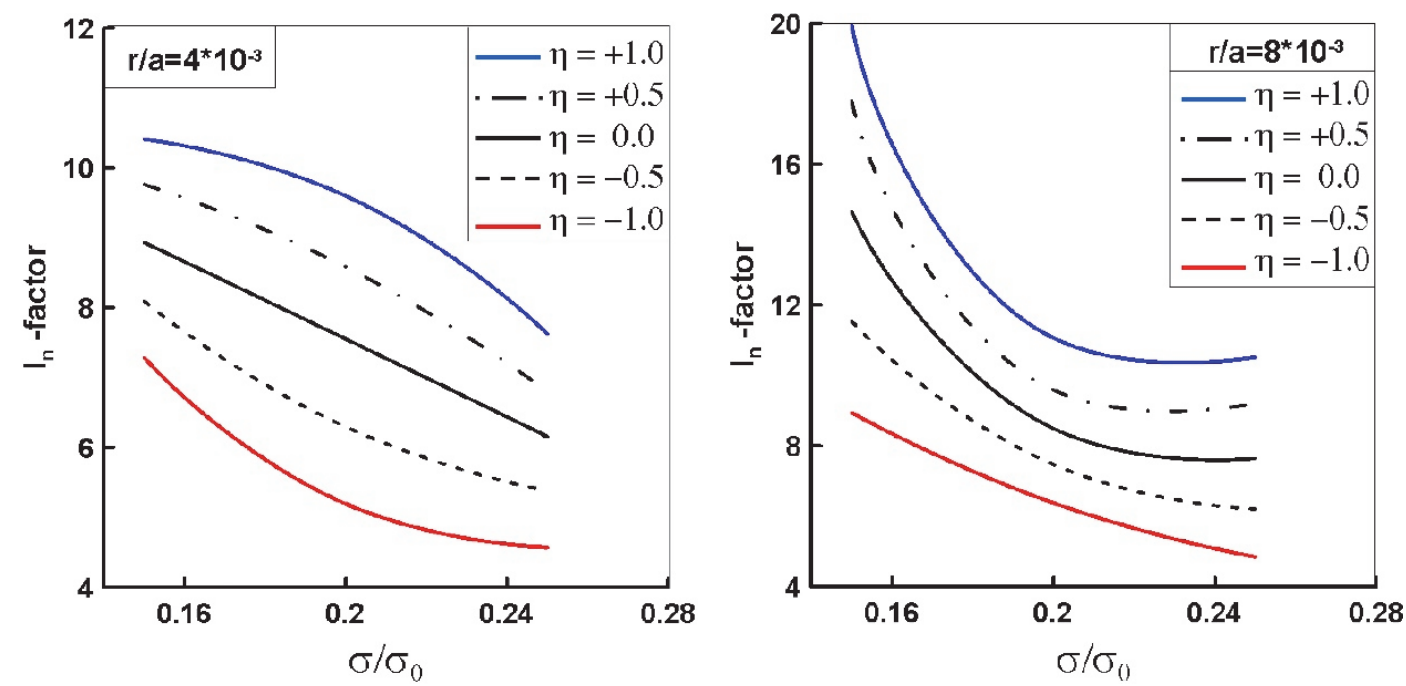

Figure 5: The governing elastic-plastic parameter distributions as a function of the applied stress level.
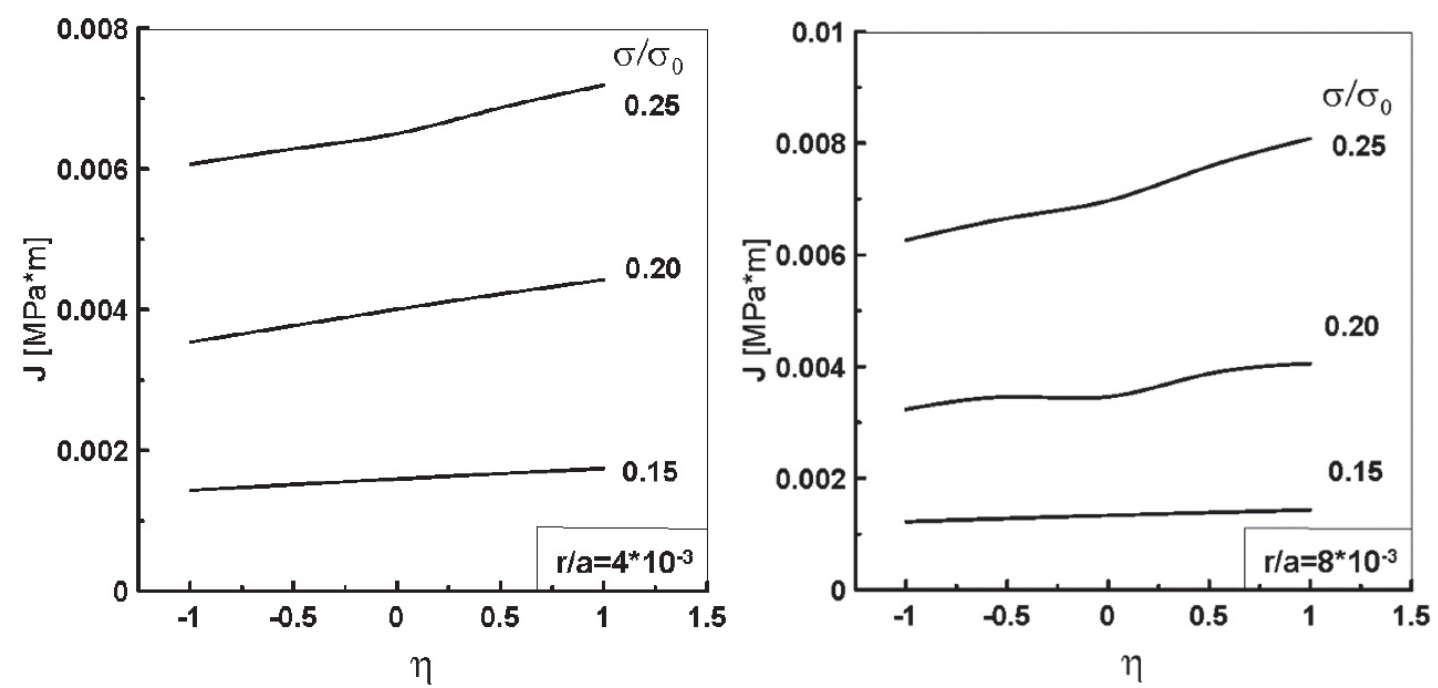

Figure 6: The $J$-integral distributions as a function of the biaxial stress ratio. 
The plastic SIF distributions as a function of the biaxial stress ratio in the range of considered the applied stress levels are given in Fig.7. Contrary to the J-integral distributions presented in Fig.6, the plastic SIF is depended on the type of the biaxial loading in the full range of the applied stresses considered here. The plastic SIF values under the equibiaxial tensioncompression $(\eta=-1)$ are higher than plastic SIF at the equibiaxial tension $(\eta=+1)$. Also it should be noted, that the plastic SIF distributions for both the crack-tip distances have the same trend.
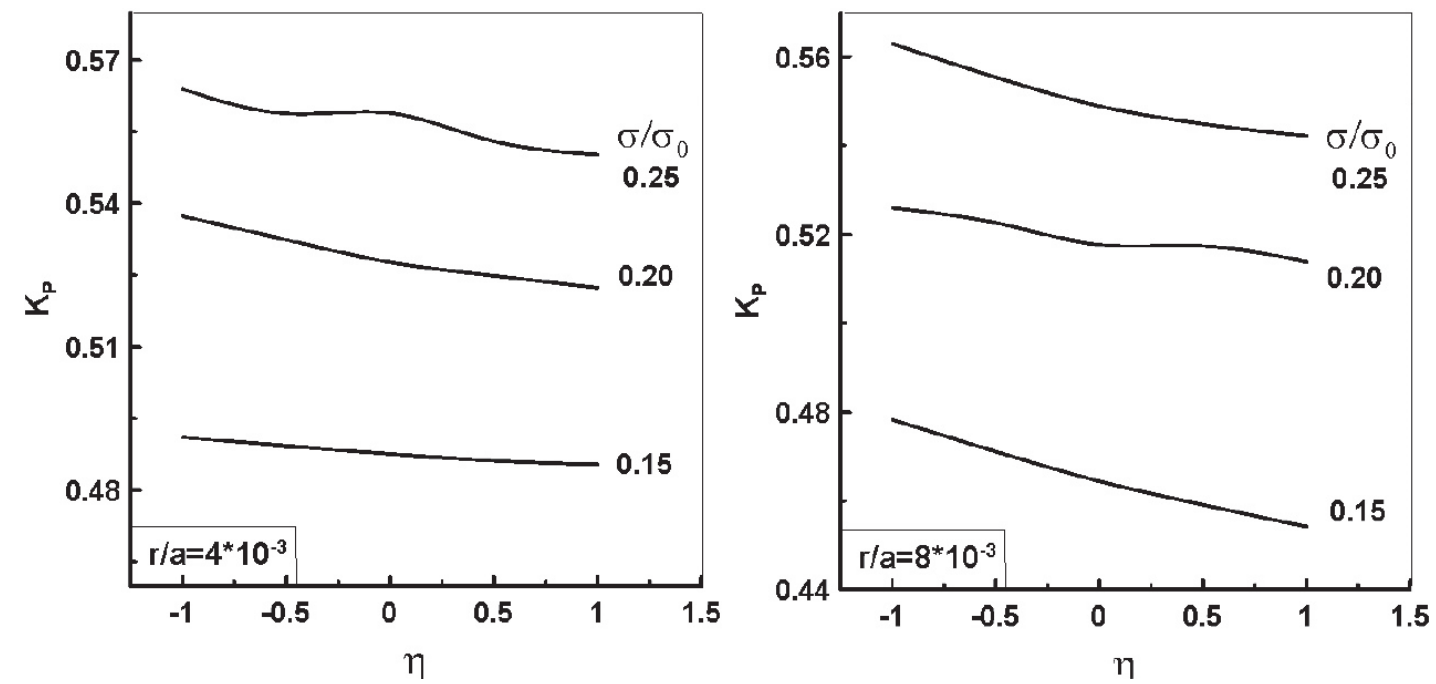

Figure 7: The plastic SIF distributions as a function of the biaxial loading.

Fig. 8 illustrates the results of comparative analysis of the values of plastic SIF calculated for the small-scale and large-scale yielding. The plastic SIF for small-scale yielding $\left(K_{s, y}\right)$ and large-scale yielding $\left(K_{p}\right)$ were calculated by Eqn.(5) and Eqn.(13) respectively. Values of the plastic SIF in Fig. 8 are presented as a function of the applied stresses for equibiaxial tensioncompression $(\eta=-1)$ and equibiaxial tension $(\eta=+1)$. In Fig.8 solid lines correspond to the plastic SIF at large-scale yielding as well as dashed lines correspond to the plastic SIF calculated for small-scale yielding.
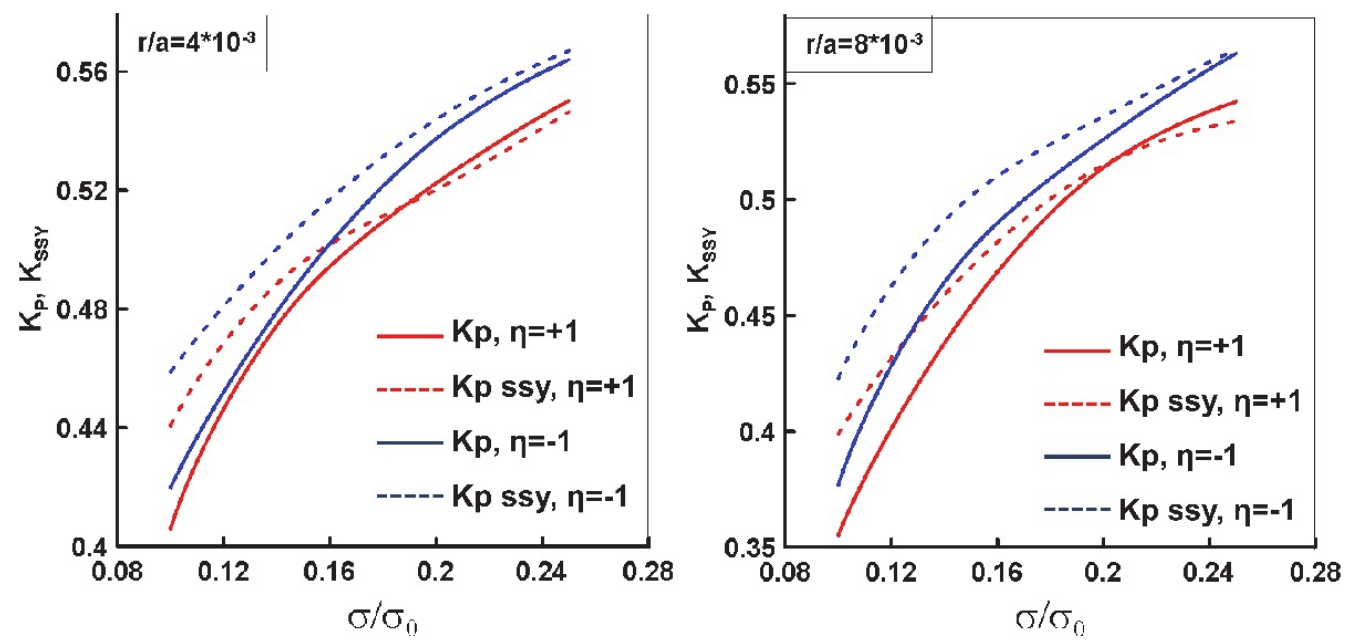

Figure 8: The plastic SIF distributions for both small-scale yielding $\left(K_{s y}\right)$ and large-scale yielding $\left(K_{p}\right)$.

In the range of the applied stresses from $\sigma / \sigma_{0}=0.1$ up to $\sigma / \sigma_{0}=0.2$ a significant difference between the plastic SIF obtained for both small-scale yielding $\left(K_{s g}\right)$ and large-scale yielding $\left(K_{p}\right)$ is observed. As the applied stresses increase, the influence of the formulation of the plastic SIF becomes insignificant. Note that the numerical results of the small-scale and the largescale yielding plastic SIF were obtained for the infinite sized central cracked plate. However, in the case of the real structures or the test specimens under complex stress state, when the plastic zone is no longer small compared to the crack length, the numerical difference between the small- and large-scale yielding plastic SIF may be more significant. The plastic SIF 
distributions for the different test specimen configurations under the mixed mode loading will be presented in the next section of this paper.

\section{MIXED MODE CRACK BEHAVIOR BY THE PLASTIC STRESS INTENSITY FACTOR}

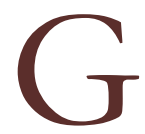

eneralization of the mixed mode crack behavior by the plastic stress intensity factor for the different test specimen configurations was presented by Shlyannikov and Zakharov [6]. Elastic-plastic parameters for the small-scale yielding such as the governing parameter of the elastic-plastic crack tip stress field $I_{n}$-factor, the stress triaxiality and the plastic SIF for the three test specimen geometries subjected to the full range of mixed mode loading were compared with the analytical solution for an infinite centre-cracked plate. The plastic SIF was calculated directly in terms of a corresponding elastic stress intensity factor using Rice's J-integral by Eqn.(5). This study is focused on the determination of the plastic SIF behavior as a function of the elastic-plastic material properties, the test specimens configuration and the mixed mode loading conditions on the base of the $J$-integral obtained by finite element method (FEM) as defined by Eqn.(11) and Eqn.(13) at the large-scale yielding range.

The elastic-plastic FE calculations were performed using the FE meshes of the cruciform and the compact tension-shear specimen configurations considered (Fig. 9) to determine the crack-tip stress-strain distributions under the different mixedmode loading conditions. To this end, the two-dimensional (2D) plane strain eightnode isoparametric elements have been used for the 2D flat CTS and CS-1 configurations, and the twenty-node quadrilateral brick isoparametric three-dimensional (3D) solid elements have been used to model the 3D biaxially loaded CS-2 with thin central part. More details of the FE meshes of the test specimens configurations considered are presented in [6].

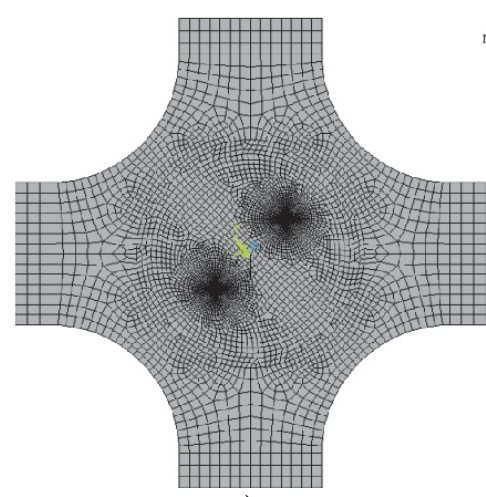

a)

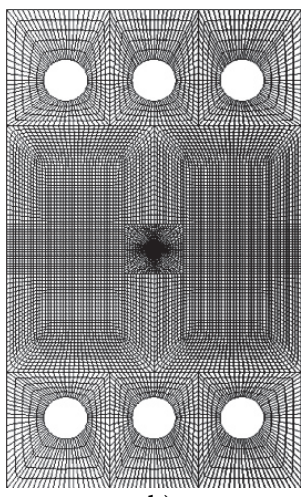

b)

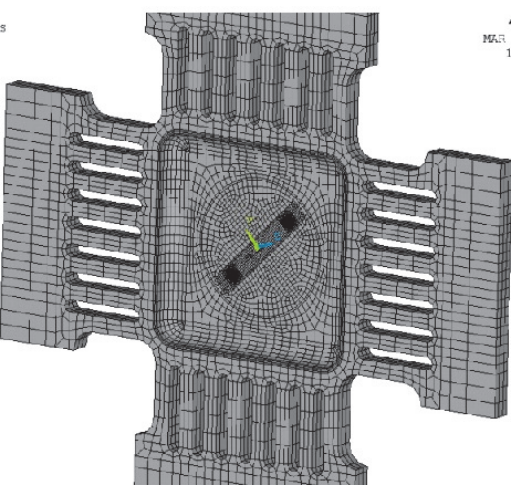

c)

Figure 9: FEM meshes of flat the CS-1 (a) and the CTS (b) geometries and 3D FEM mesh of the CS-2 (c) specimen.

As mentioned above, the CS-1, CS-2 and the CTS configurations exhibit a full range of the mixed-mode fracture conditions for plane strain and the full-field 3D problems due to the different values of the combination for the crack inclination angle $\beta$ and the loading biaxiality $\eta$. The FEM numerical solutions have been obtained for a large number of the different combinations of the crack inclination angle and the nominal stresses $\sigma_{n}$ in the considered test specimen geometries. The different degrees of the mode mixity from pure Mode I to the pure Mode II were characterized by a near-field mixity parameter introduced by Shih in [2]. For the mixed-mode small-scale yielding problems the mixity parameter can be expressed in the following form:

$$
M_{p}=\frac{2}{\pi} \tan ^{-1}\left|\frac{\tilde{\sigma}_{\theta \theta}(\theta=0)}{\tilde{\sigma}_{r \theta}(\theta=0)}\right|
$$

where: $\theta$ - the polar angle, $\tilde{\sigma}_{\theta \theta}, \tilde{\sigma}_{r \theta}$-the dimensionless functions of the stresses. It should be noted that $M_{p}=0$ corresponds to the pure Mode II and the pure Mode I is realized when $M_{p}=1$.

Fig. 10 shows the distributions of the $J$-integral for all considered the test specimen configurations with the set of the elasticplastic materials properties as a function of the mode mixity $M_{P}$ ranging from 0 to 1 . As it follows from these results, the elastic-plastic material properties has the significant effect on the $J$-integral distributions in a full range of the mixed modes. 
It should be noted that the J-integral at pure Mode I is less than that at pure Mode II. It can be explained in terms of the plastic zone that develops directly ahead of the crack tip in a large scale under a mixed mode loading with respect to pure Mode I.

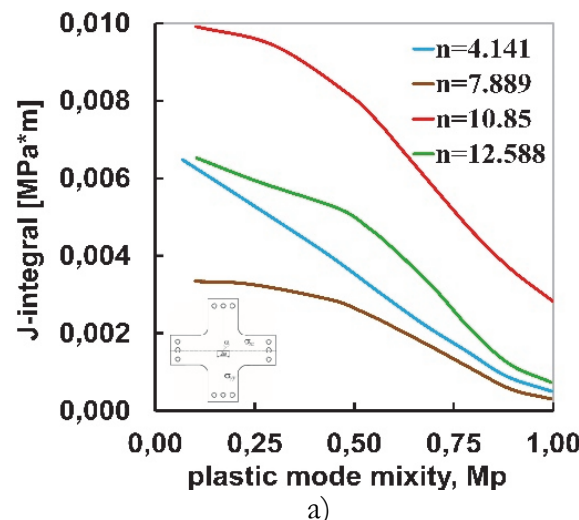

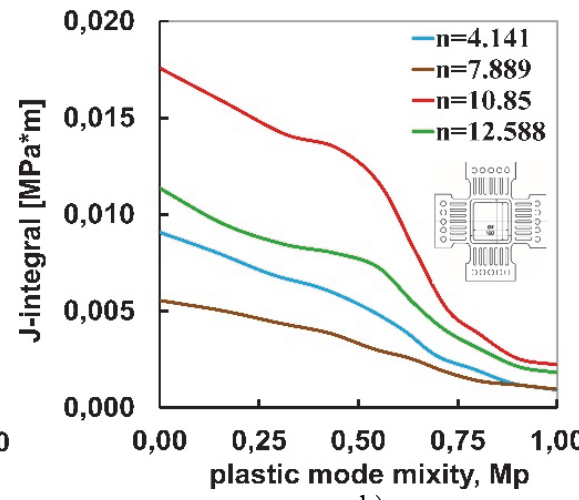

b)

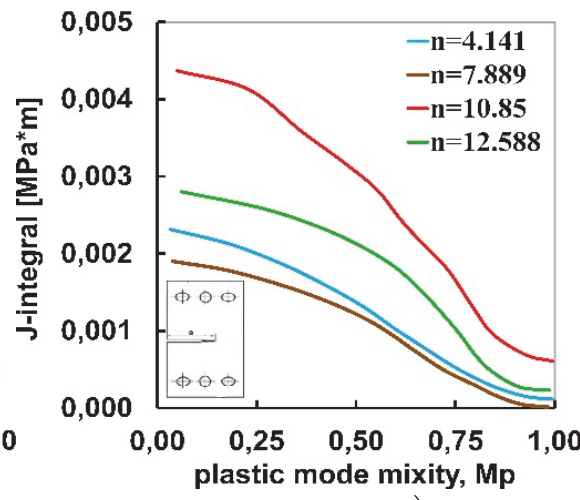

c)

Figure 10: The effect of material properties on the J-integral for the CS-1 (a), the CS-2 (b) and the CTS (c).

The results of the J-integral behavior are shown in Figs. 11a, 11b, 11c and 11d for mixed mode conditions for the four materials corresponding to the strain hardening exponent $n=4.13$ (Steel P2M), $n=7.89$ (Steel 34Cr), $n=10.85$ (Al-alloy $7050)$ and $n=12.59$ (Ti6Al4V), respectively. These results illustrate the effects of mixed mode fracture for each material considered for a set of the specimen configurations. As it follows from these results, the influence of the test specimen geometry on the behavior of $J$-integral is observed in the full range of the mixed mode loading.
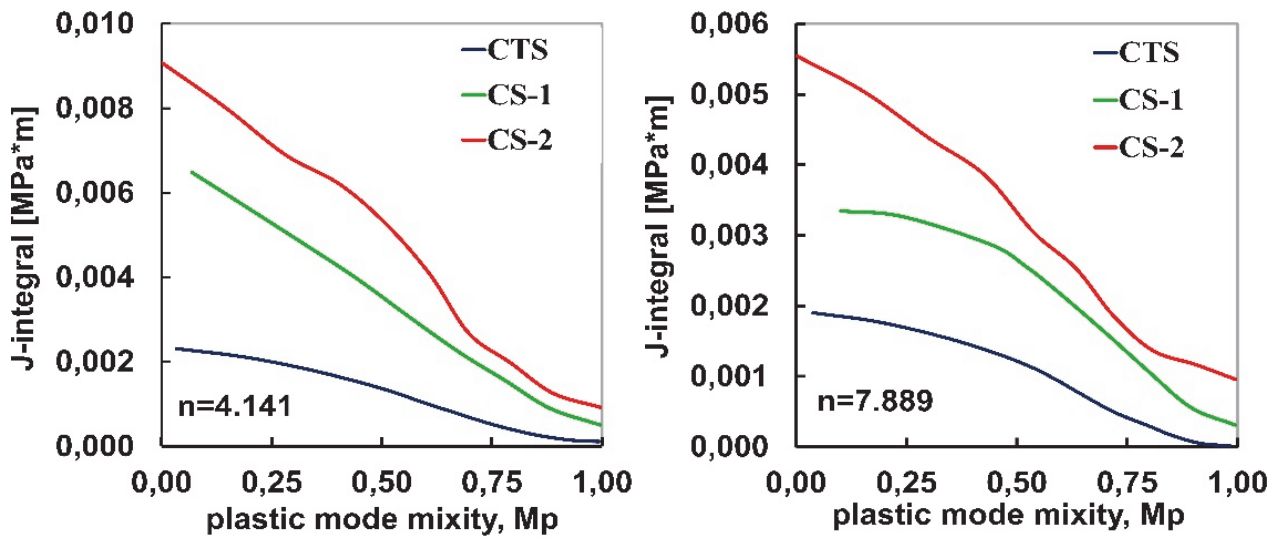

a)

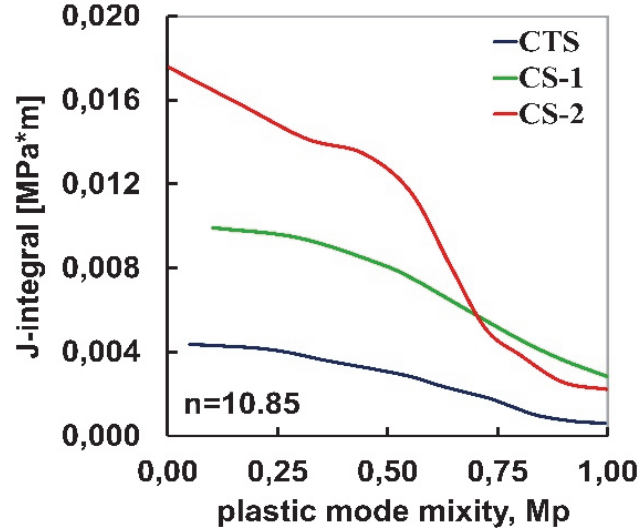

c)

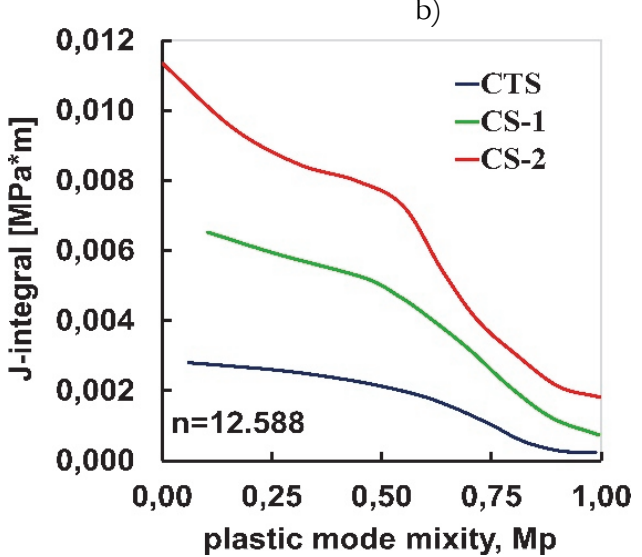

d)

Figure 11: Variations of the $J$-integral for the test specimens as a function of the material properties. 
On the base of the numerical solutions for the $I_{n}$-integral and the $J$-integral defined as Eqn.(8) and Eqn.(11) respectively, the plastic SIF distributions for all test specimens considered were obtained as a function of the material properties, described by the strain hardening exponent $n$ and the mode mixity $M_{p}$ ranging from 0 to 1 . Note that the plastic SIF was calculated for the large-scale yielding approach by Eqn.(13).

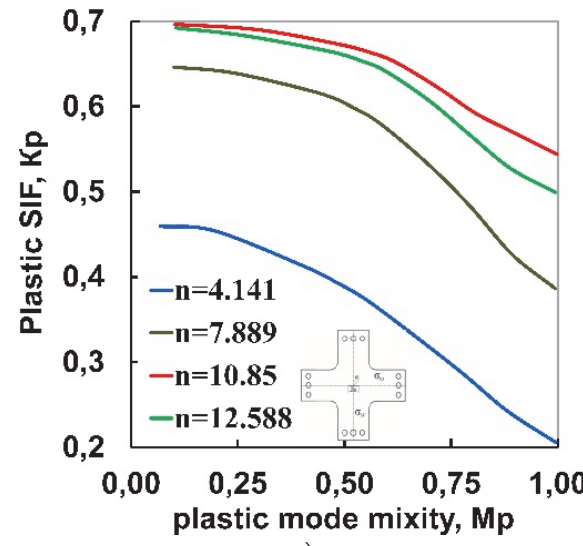

a)

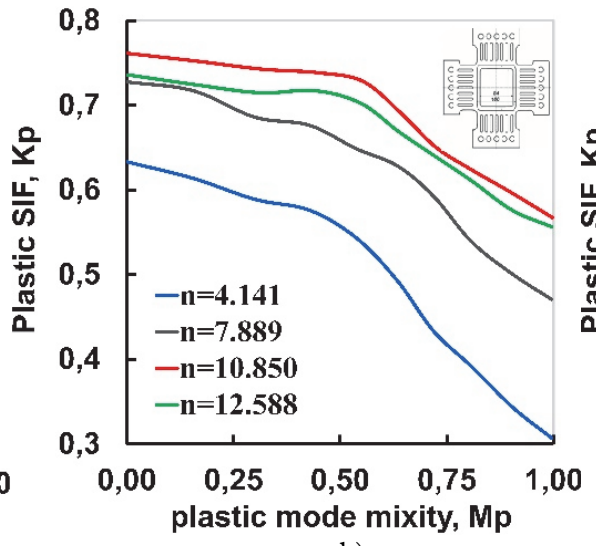

b)

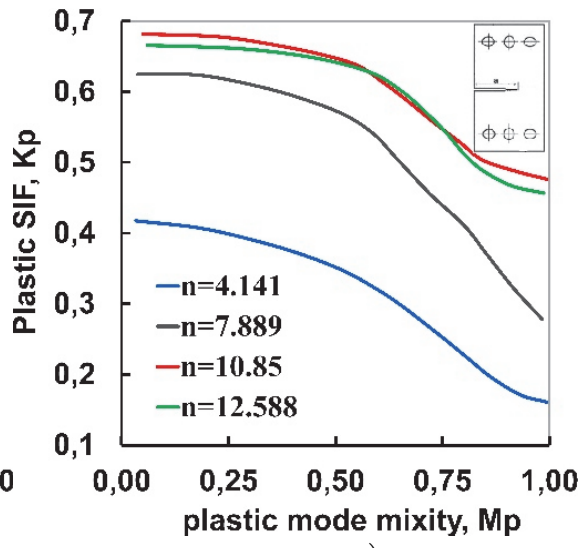

c)

Figure 12: The effect of the material properties on the plastic SIF distributions.

The results presented in Fig.12 illustrate a sensitivity of the plastic SIF to the elastic-plastic material properties and the loading conditions. It should be noted that for all test specimens configurations the plastic SIF is increased with increasing of the strain hardening exponent $n$.
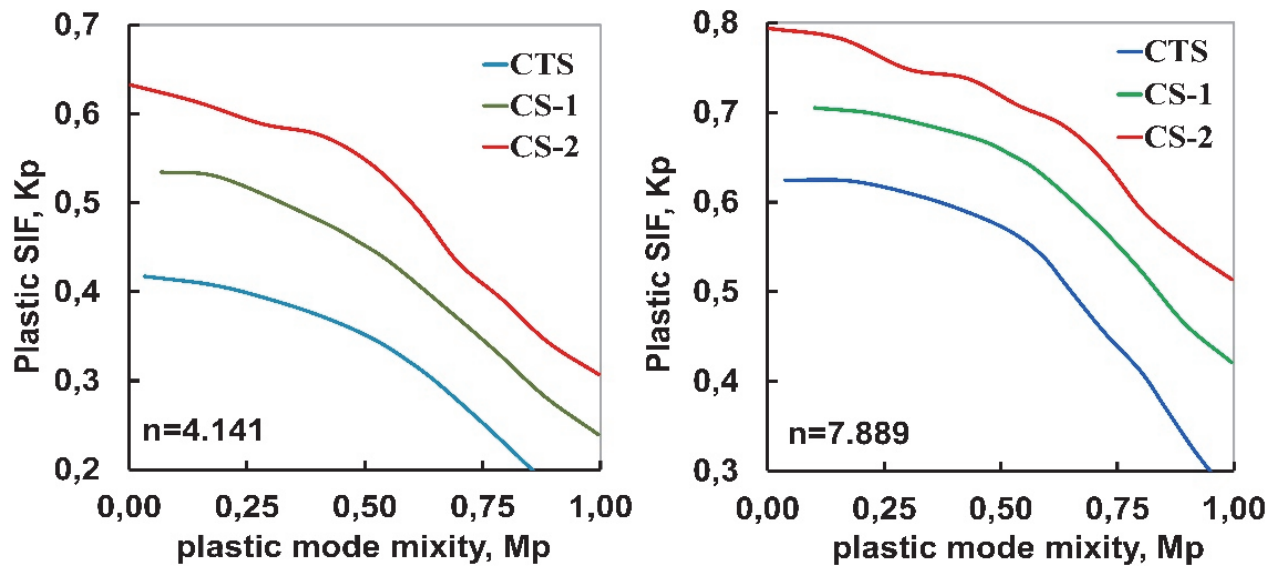

a)

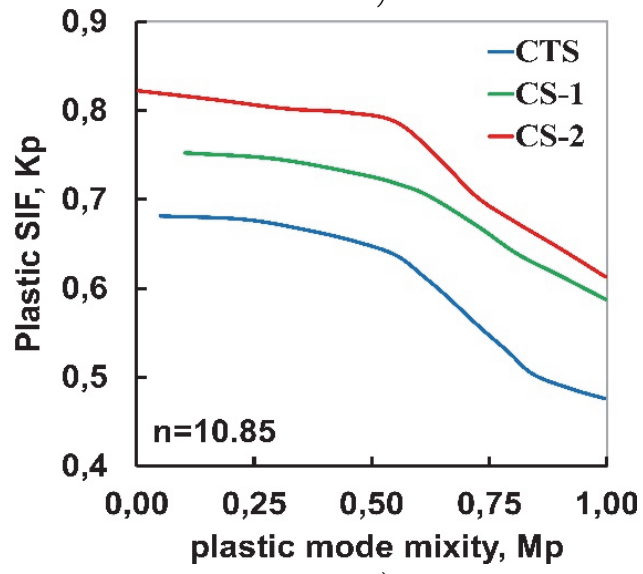

c)

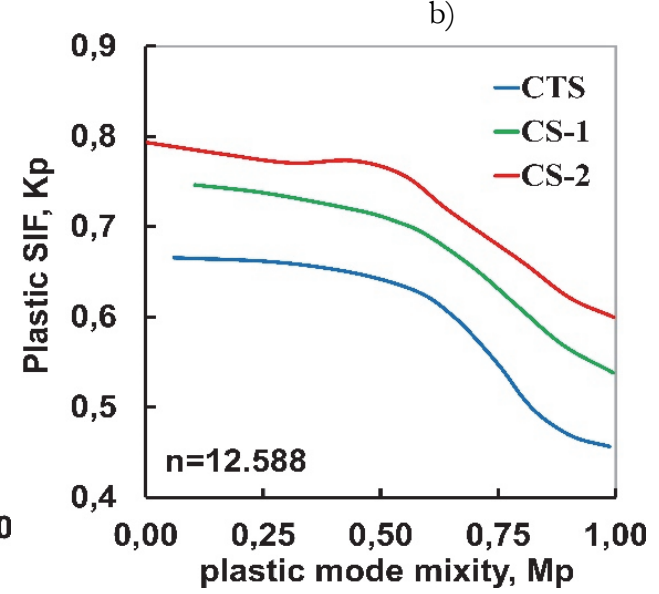

d)

Figure 13: Distributions of the plastic SIF for the test specimens as a function of the material properties. 
Influence of the test specimen configuration on the plastic SIF distributions in full range of the mixed modes of fracture is presented in Fig.13. In the full range of the mixed mode loading from the pure shear $\left(M_{p}=0\right)$ up to the normal tension $\left(M_{p}\right.$ $=1$ ) the monotonic a decreasing of the plastic SIF is observed.

Comparative analysis of the values of the plastic SIF calculated for both small-scale and large-scale yielding in the test specimen configurations considered here is presented in Fig.14. Note, that plastic SIF for the small-scale yielding $\left(K_{s y g}\right)$ and the large-scale yielding $\left(K_{p}\right)$ were calculated by Eqn.(5) and Eqn.(13) respectively. Values of the plastic SIF on Fig.14 are presented as a function of the mixed mode loading for high strength steel with the strain hardening exponent $n=4.141$ and titanium alloy $(n=12.59)$. In Fig.14 the solid lines correspond to the plastic SIF at large-scale yielding as well as the dashed lines correspond to the small-scale yielding plastic SIF.

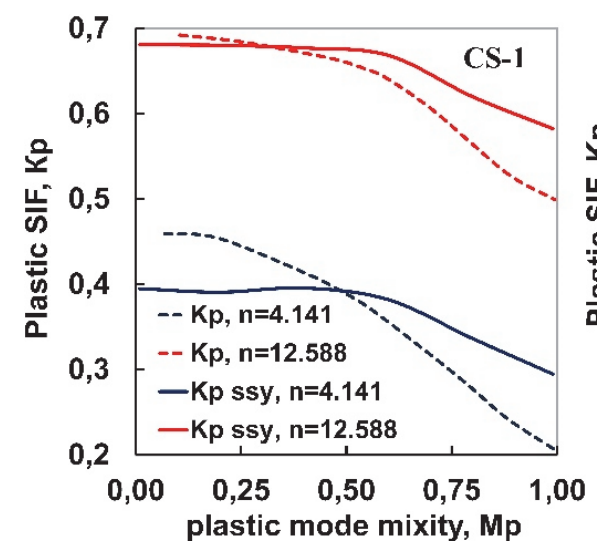

a)

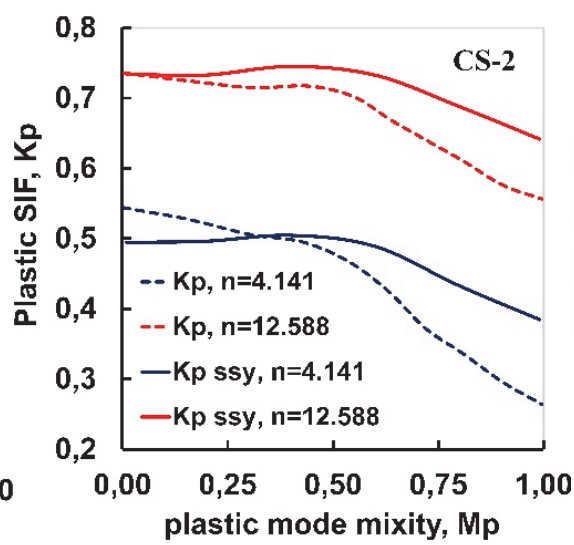

b)

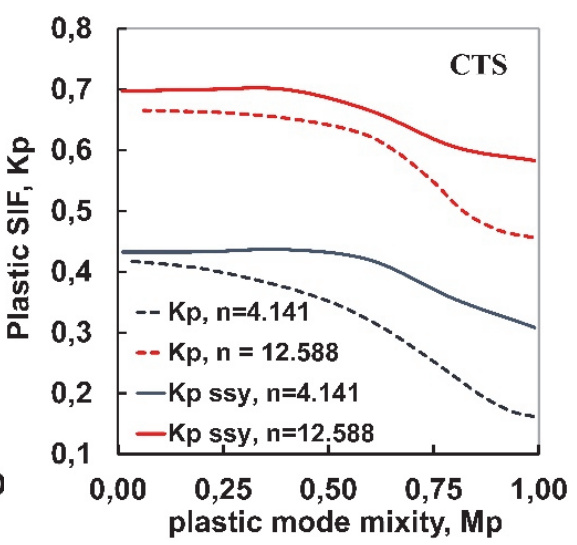

c)

Figure 14: The plastic SIF distributions for both small-scale yielding $\left(K_{s g}\right)$ and large-scale yielding $\left(K_{p}\right)$ for the CS-1 (a), the CS-2 (b) and the CTS (c).

As it follows from the results presented in Fig.14, there is a significant difference between the small- and the large-scale yielding plastic SIF in the full range of mixed modes. Therefore in the case of cracked bodies under mixed mode loading it is not correct to use the plastic SIF formulation in accordance with Hutchinson's and Shih's relations [1, 2] between the Jintegral and $K_{p}$ in the form of Eqs. 2-4. To avoid increasing the differences in the values of the plastic SIF in a full range of mixed-mode nonlinear deformation, one can use Lee and Liebowitz's algorithm [10] for numerical determination of the Jintegral under large-scale yielding.

\section{CONCLUSIONS}

he infinite sized central cracked plate under biaxial loading as well as the cruciform specimens of two configurations and the compact tension-shear specimen subjected to mixed Mode I/II loading were used to study the crack-tip fracture resistance parameters by using an elastic-plastic FE analysis. Coupling effects of the biaxial stress ratio and the applied nominal stresses on the $J$-integral and governing parameter of elastic-plastic crack-tip stress fields $I_{n}$-integral as well as the plastic stress intensity factor behavior were stated. Values of the plastic stress intensity factor were calculated by using small- and large-scale formulation. For infinite sized central cracked plate significant difference between small-scale yielding $\left(K_{s y}\right)$ and large-scale yielding $\left(K_{p}\right)$ was observed in the range of applied nominal stresses up to 0.2 , then as the applied stresses increase, the influence of formulation of the plastic SIF becomes insignificant. Special emphasis was put on the behavior of the J-integral and the plastic SIF for specified test specimen geometries under mixed mode loading. For all considered test specimen configurations trends of the $J$-integral as well as the plastic stress intensity factor behavior as a function of mode mixity and material nonlinearity were founded. The applicability of the plastic stress intensity factor approach to large-scale yielding analysis of cracked bodies under mixed mode loading was demonstrated.

\section{ACKNOWLEDGMENT}

$\mathrm{T}$ he authors gratefully acknowledge the financial support of the Russian Science Foundation under the Project 1979-10160. 


\section{REFERENCES}

[1] Hutchinson, J.W. (1968). Singular behaviour at the end of a tensile crack in a hardening material. J. Mech. Phys. Solids., 16, pp. 13-31. DOI: 10.1016/0022-5096(68)90014-8.

[2] Shih, C.F. (1974). Small-scale yielding analysis of mixed mode plane-strain crack problems. ASTM STP, 560, pp. 187210. DOI: $10.1520 /$ STP33141S.

[3] Shlyannikov, V.N., Tumanov, A.V. (2014). Characterization of crack tip stress fields in test specimens using mode mixity parameters. Int. J. Fract., 185, pp. 49-76. DOI: 10.1007/s10704-013-9898-0.

[4] Shlyannikov, V.N., Boychenko, N.V., Tumanov, A.V., Fernandez-Canteli (2014). A. The elastic and plastic constraint parameters for three-dimensional problems. Engng. Fract. Mech., 127, pp. 83-96. DOI: 10.1016/j.engfracmech.2014.05.015.

[5] Shlyannikov, V.N., Tumanov, A.V., Zakharov, A.P., Gerasimenko, A.A. (2016). Surface flaws behavior under tension, bending and biaxial cyclic loading. Int. Journ. Fatigue., 92, pp. 557-576. DOI: 10.1016/j.ijfatigue.2016.05.003.

[6] Shlyannikov, V.N., Zakharov, A.P. (2017). Generalization of mixed mode crack behavior by plastic stress intensity factor. Theoret. Appl. Fract. Mech., 91, pp. 52-65. DOI: 10.1016/j.tafmec.2017.03.014.

[7] Hilton, P.D., Hutchinson, J.W. (1971). Plastic intensity factors for cracked plates. Eng. Fract. Mech., 3, pp. $435-451$. DOI: 10.1016/0013-7944(71)90057-9.

[8] Hilton, P.D., Sih, G.S. (1973). Applications of finite element method to the calculations of stress intensity factors. Mechanics of Fracture. Methods of Analysis and Solution of Crack Problems, 1, pp. 426-483.

[9] Hilton, P.D. (1973). Plastic intensity factors for cracked plates subjected to biaxial loading. Int. J. Fract., 9, pp. 149_ 156.

[10] Lee, J.D., Liebowitz, H. (1977). The nonlinear and biaxial effects on energy release rate, J-integral and stress intensity factor. Engng. Fract. Mech., 9, pp. 765-779. DOI: 10.1016/0013-7944(77)90002-9.

[11] ASTM Standard E8-04. (2004) Standard test method tension testing of metallic materials. Annual Book of ASTM standards., Amer. Soc. Test. Mater., 01.02. 14.

[12] Rice, J.R. (1968). A path independent integral and the approximate analysis of strain concentration y notches and cracks. ASME J. Appl. Mech., 35, pp. 379-386.

[13] ANSYS. (2009). Theory Reference for the Mechanical APDL and Mechanical Applications. Release 12.0. Available at: http://dl.mycivil.ir/reza/ans_thry.pdf. 